\title{
Three Models of Equivalence in English Translation of Chinese Classics in the Light of the Theory of Translation as Selection and Adaptation
}

\author{
Guodong Zhao \\ Foreign Languages Department, Inner Mongolia Finance and Economics College, Huhhot, Inner Mongolia, China \\ Email: godwin1116@sohu.com \\ Xiao Liu \\ Foreign Languages Department, Inner Mongolia Finance and Economics College, Huhhot, Inner Mongolia, China \\ Email: zhaoliu1116@163.com
}

\begin{abstract}
Many researches have been done to justify that equivalence is the supreme target of translation. However, so far, no research has ever figured out some applicable methods as to how to achieve "equivalence". This paper, based on the theory of "Functional Equivalence" and the Theory of Translation as Selection and Adaptation (TASA), formulates three ways of translation for the translation of Chinese classics into English, namely, integrated model, style oriented model and information oriented model.
\end{abstract}

Index Terms - equivalence, TASA, Chinese classics, translation models

\section{INTRODUCTION}

English Translation of Chinese Classics is much demanding due to the distinct differences between the two languages structures and the heavily loaded cultures as well. Equivalence between the source text and target text has long been the pursuit of many translators and translation theorists, but such equivalence is only an ideal. This paper is dedicated to work out specific guidelines - some workable translation models based on the principle of adequacy and acceptability in translation - concerning English translation of Chinese classic novels.

\section{LITERATURE REVIEW}

In history, many scholars have put forward many standards for translation practices. Alexander Fraser Tytler (2007) raises three translation principles in the essay On the Principles of Translation, which can be generalized as "a translation should give a complete transcript of the ideas of the original work; the style and manner of writing in a translation should be of the same character with that of the original; a translation should have all the ease of original composition".

The three source text oriented principles formed the cornerstone of western translation theory. Nida's theory (1964) - dynamic equivalence - emphasizes the response of the readers of the source text in the source culture. Later the German translator Juliane House (1977) gives a definition of functional equivalence in terms of sociolinguistic dimensions in the source text. So, these scholars all regard equivalence as a matter of quality of translation. As to either Nida or House, the assessment of translation only concerns the determination of equivalence between the translation and the source text. They both neglect the possible position of translation as a member of target literary system, as text among texts in the target culture. Peter Newmark (1980) states that the meaning of the source text gets lost due to over-translation or inadequate-translation. His statement emphasizes that equivalence in translation is a matter of degree rather than a state. Hu Gengshen (2006) also believes there is no absolute equivalence between the source text and target text. The so called equivalence is only the closest match to the source text in form, style and function. Translation, in his opinion, is a selection among many alternatives, and the best selection should fit the translation environment consisting of many factors such as the authors, the source text, the source culture, the source language structure, the target readers, the target text, the target language and culture. This theory is applicable for it puts the translators at the center of the whole translation practice and emphasizes translation as a multi-dimensional selection and adaptation. This paper tries to figure out a translation model for the English translation of Chinese classic novels based on the above mentioned theories and the translation of The Outlaws of the Marsh and the Dream of Red Mansion.

\section{RESEARCH METHODS AND SCHEME}


By analyzing different translation situations with regard to different texts, this paper classifies the texts into three types, namely style oriented text, information oriented text and ordinary text which centers the style and information equally. Based on different texts the paper deducts three translation models theoretically, i.e. style oriented model, information oriented model and the integrated model, which all should take functional equivalence as the final target. In order to illustrate the three models, the paper raises some example to show the application of the models in the translation of Chinese classics into English.

The first part of the paper is the introduction. Part two deals with the literature review, aiming to explain the concerned translation theories in history, and attempts to work out the problems remained. Part three is about the research method and scheme of the paper. Part four explains the relationship between the meaning, style and function in a text, and accordingly raises three models. Part five is the illustration of the three models with case study. Part six draws a conclusion.

\section{Applicable Models of Translation}

\section{A. The Interrelationship between Meaning, Style and Function in Translation}

The classic novels like The Outlaws of the Marsh and A Dream of Red Mansion are the representatives of ancient Chinese literature. They contain a rich and colorful cultural heritage. Both the language structure and the style are heavily culture-loaded. The meaning, style and the function of the language are as a whole in the source text. The meaning of the text lies in the style of the language, and the special style of the language in turn fulfills certain functions, and the function of the language is intermingled with the meaning. But anyway, no matter what kind of style the text is manifested in, it must emphasize certain functions.

Jakobson (1960) establishes a framework of language functions based on six key elements of communication, namely referential function, poetic function, emotive function, conative function, phatic and metalingual functions. Accordingly, any language form must have one or few of these functions and the referential function which mainly focuses on the semantic aspect of the language, is only one of many functions that language can achieve. No matter what kind of function it is, it must be achieved through the use of certain style, whether plain or special. Halliday (2000) makes it even simpler, and he states that there are basically three functions of language: ideational, interpersonal and textual. The functional translation theorist Katharina Reiss (1971) points out that the functions of the text should be listed as one of the standards by which to judge a translation work, and the basis of the standard is supposed to be the relationship between the functions of the source and target text. That is people can judge a translation work by the relationship between the functions of the source text and the target text.

\section{B. Adequacy in Understanding the Source Text and Acceptability in Target Text}

So far this paper has discussed in the above sections the relationship between the style, meaning and functions of a text. It can be easily concluded that the three factors, the meaning, style and function of a specific text can not be treated in isolation. However, a specific text does not totally possess the three factors equally, rather, it has certain emphasis. Sometimes, the text mainly contains some information, and sometimes it pays due attention to the interpersonal functions it can achieve, and in very few cases, the text is applied for the sheer purpose of amusing and the style is the most emphasized factor. A good translator needs to have a deep understanding of the text and tries to figure out what the source text is oriented. If equivalence can be achieved, it is only equivalence of the possibly emphasized factors between the source and target text. An adequate understanding is very demanding to the translators. The translator needs to grasp the purposes of the author in the source text, and needs to be sensitive to the differences between the two languages and cultures. Then a rational selection can be made based on such knowledge.

The next step goes to the organization of the target text. By acceptability I mean that the target text should to a large extend fit the author's purpose in composing the source text, the major functions of the source text, the style and the function of the style in the source text, the equivalence possibilities between the source and target texts, the habit of expression of the target, which as a whole is what Hu Gengshen (2006) called the translation ecological environment. If there is no absolute equivalence between the source and target text, a translator's work should be focused on striving for selections among possible alternatives. This selection is supposed to be made based on: an adequate understanding of the source text; an overall consideration of the translation environment (an ecological system consisting of the source language, source text, source culture, target language, target text, target culture, the target readers, etc); acceptability of the target text among the target readers.

\section{Models of Translation}

What has been discussed above comes to the conclusion that there is seldom complete equivalence between the source text and target text. The absolute equivalence is nothing but an ideal. It is better to use the term "functional equivalence" to describe the range of adequacy and acceptability in translation. Just as Nida put it: "the equivalence means the readers of a translated text should be able to comprehend it to the point that they can conceive of how the original readers of the text must have understood and appreciated it" (Nida, 2001)

So, if there is no complete equivalence in translation, the translator's major job would be striving for the closest match in the target text concerning functions. As to the actual translation, there are basically three models to follow. 


\section{a. An Integral Model}

The first model is the most common one. Equivalence can be achieved totally from the three factors, namely, style, meaning and function. The basis for this model is as following: (1) The style applied in the source text has a closest match in the target text, and the style in both texts has similar or identical functions. (2) The meaning conveyed in the source text can be expressed in the target text with the style mentioned in (1). (3) Both the style and meaning in the target text can contribute to the fulfillment of the functions that the original work aims to achieve. If perceived in the light of the theory of translation as selection and adaptation proposed by Hu Gengshen, (Hu Gengshen: 2006) the selection is made in style, meaning and functions totally, and such a selection is most adaptable one in the translation ecological environment and accordingly, the effect of the translation in target environment is identical with what it is in the original. In this case, equivalence is gained both in form and in function. As an integrated unit, the three factors of the original text are totally transferred to the target text. The translated version is not only a good match of the original but is well accepted by the target readers.

To be noted is this model is different from literal translation. Literal translation does not regard the style and meaning as a whole, or as an integrated unit. It will either transfer the style merely or the meaning in isolation. The result is that the translated version can not have identical functions as the original version has. However, this model emphasizes the interrelationship between the style and the meaning it conveys, and emphasizes the same functions to be achieved in both languages and both cultures. It is called an integrated model in that it treats the three factors of the text as a whole in translating process.

\section{b. Style Oriented Model}

If the original text focuses on the style rather than the literal meaning of the words or sentences to achieve certain functions, the translation of the text is supposed to center on the style of the text to achieve similar functions in the target text on the condition that equivalence can be obtained at the level of style. In this case, the literal meaning in both the original and target texts is superficial, and it serves the expression of certain function by means of particular style of the language. So the literal meaning can be shifted in the process of translation while the functions can not. If the closest match can be found concerning style, a style oriented model is applied.

We know that the complete equivalence is an ideal state of translation. However, when such equivalence can not be achieved, translators need to make selection among the three factors. The selection is the best if it can to the greatest extent has the same function and effect as the original text has. A style oriented is a best alternative giving that (1) the literal meaning is not substantial and (2) the main function is achieved by means of the style, and (3) there is the closest match in style in target language.

c. Information Oriented Model

Yanfu once put forward three difficulties in translation "faith, readability, and elegance." Nida (1974) points out that translation means to re-produce the closest and most natural expression of the source information in semantics and style in the target text. Wang Dongfeng (2004: 9) once explains "being faithful" as "being close to" rather than "being identical". Yanfu's theory is supreme guideline of translation activity, but still, "faith" does not mean "being identical". If the original text is not elegant, the translated version should not be elegant; otherwise the standard of being faithful will be violated. Nida emphasizes information equivalence in translation, and he agrees that such equivalence is a matter of degree. The information oriented model of translation is a compromise made in the target language in that the aesthetic effect and style of the original text could hardly find a match in the target language due to linguistic and cultural differences. For instance, Chinese is rich in idioms consisting of four Chinese characters. Theses idioms are condense in meaning and regular in form. The aesthetic effect is special in context. Furthermore, the meaning contained in the idioms is very deep and heavily cultural loaded. There are seldom English matches to these idioms. In translation, an information oriented model is better applied than others. To be exact, in translation, translators tend to interpret but not to find the match.

Based on the above discussion, I conclude that an information oriented model is applied under the condition that (1) heavily culture loaded style of the source text has no match in the target language; (2) the aesthetic effect of the source text can not be similarly achieved in the target language; (3) the major purpose of the source text is to convey some information through the use of the unique style of the language.

\section{CASE STUdy}

So far, the paper has worked out three tentative translation models. They are all micro methods concerning specific translation work. In translation practice, many translators consciously or unconsciously follow these guidelines. Shapiro's translation of the Chinese classic novel The Outlaws of the Marsh and the different translation versions of A Dream of Red Mansion, Monkey King and Romantic of Three Kingdoms and other novels can well illustrate the three models above. For example:

Eg. 1 linChong chi le ba jiu bei jiu, jiu yao xiao yi, qi shen dao: "wo qu jing shou le lai". Lin Chong xia dei lou lai, chu jiu dian men, tou dong xiao xiang zi qu jing le shou. (Chinese version)

Lin Chong downed eight or nine cups. Soon he had to relieve himself. He got up and said, "I have to wash my hands". He went downstairs, left the tavern, and attended to his business in a small lane to the east. (Outlaws of the Marsh chapter 7 translated by Shapiro) 
Eg. 2 WuYong xiao dao: "bu xu xiong zhang gua xin, WuMou zi you cuo zhi, zi gu dao: 'shui lai tu yan, bing lai jiang ying,"'. (Chinese version)

The teacher laughed. "There is nothing to worry about. I have a plan. Earth can stop the flow of water, generals can cope with the enemy troops." (Outlaws of the Marsh chapter 20 translated by Shapiro)

Eg. 3 Pu di zhi yi quan, zheng da zai bi zi shang, da de xian xue beng liu, bi zi wai zai ban bian, que si kai le ge jiang you pu, xian de, suan de, la de, yi fa dou gun chu lai. (Chinese version)

He landed a punch on Zheng's nose that flattened it to one side and brought the blood flowing like sauces in a condiments shop - salty, sour, and spicy. (Outlaws of the Marsh chapter 3 translated by Shapiro)

In example 1, the Chinese expressions "xiaoyi" and "jingshou"are both used as euphemism. Both the style and the meaning have equal expressions "relieve himself", "wash my hands", and "attended to his business" in English, which is the basis of literal translation. The style and the meaning thus can be treated as an integrated unit in translation and be transferred directly to the target text. Example 2 indicates the translation of some proverbs can be done literally with the style and meaning kept similarly in the target text. The reason for this kind of translation is that although there is no absolute equivalence in style in English, direct transferring of the original style can still be understood and accepted by the target readers. An integrated model is applicable in that the flavor of the original version can be simultaneously felt in the target text. Example 3 represents a common phenomenon in translation. Most of the similes used in Chinese can be treated directly in English translation, for superficial similarity can be similarly felt by any people in the world, which reflects the common cognition of the world of human beings. In addition, the three English words "salty, sour, and spicy" vividly depict the situation on that occasion. The three words form the most suitable correspondence to the source text "xian de, suan de, la de". So, the above three examples show that in translation the translatable elements style and meaning are selected as an integrated unit to deal with. And such selection is the most suitable one in that the target text is adaptable to the target language environment and can fully express the functions of the original text. But the following example is different and the second model is applied.

Eg. 4 er ren zheng shuo zhe, zhi jian Xiang Yun zou lai, xiao dao: “ai ge ge, Lin mei mei, ni men tian tian yi chu wan, wo hao bu rong yi lai le ye bu li woer". DaiYu xiao dao: "pian shi yao she zi zi ai shuo hua, lian ge er ge ge ye jiao bu chu lai, zhi shi 'ai' ge ge, 'ai' ge ge de, hui lai gan wei qi, you shuo ni nao yao 'ai' san le”.

"couthin Bao, couthin lin: you can thee each other everyday. It'th not often I get a chanthe to come here; yet now I have one, you both ignore me!" Dai-yu burst into laughing: "lisping does not seem to make you afraid of talking! Listen to you: 'couthin', 'couthin'! Presently, when you're playing Racing Go, you'll be all 'thicktheth' and 'theventh'!" ( $A$ Dream of Red Mansion translated by David Hawkes)

In the novel, Shi Xiang-yun is depicted as lisping. By using two pronunciations "er" and "ai", means the second in Chinese, the Chinese version vividly portrayals a lisping girl. Here, the meanings of both "er" and "ai" are not emphasized, however the style of the two is typical. In order to reflect the functional style of the source text, Hawkes purposefully chooses two consonants in English /s/ and $/ \theta /$. So, the counterparts of the words like "Bao Gege," (cousin Bao) and "Lin Jiejie,"(cousin Lin ) in English are replaced by "couthin Bao" and "cousin Lin". The words uttered by Xiang-yun such as "see, chance, sixth, seventh" become "thee, chanthe, thichtheth, theventh". Thus, a lisping girl's feature is vividly presented on the paper for readers to appreciate. By using English consonants to replace the Chinese vowels, the translator makes the functions of the target text equals that of the source text with regard to the styles. So in this example, it is the style that is selected as the major point in translation. It is not necessary to translate the source language word by word, but the style is strictly kept in the target text.

The third model is used when the style is plain but the meaning or information conveyed is more important. Let's look at the following few sentences.

Eg. 5 Ning rong liang fu shang xia nei wai ren deng, mo bu huan tian xi di. (Cao Xueqin A dream of Red Mansion Chapter16)

Then high and low alike in both mansions were filled with joy. (Translated by Yang Xianyi)

Eg. 6 Na xiao si huan tian xi di, da ying qu le. (Cao Xueqin A dream of Red Mansion Chapter 39)

The boy assented and made off in high spirits. (Translated by Yang Xianyi)

Eg. 7 liang ge xiao tou mu ting le zhe hua, huan tian xi di, shuo dao: "hao le, zhong ren zai ci shao dai yi shi"

Nai'an The Outlaws of the Marsh Chapter 17)

The junior officers were delighted. "Excellent," they said. "Just wait here a little". (Translated by Shapiro)

Eg. 8 De meng cai qian, huan tian xi di, shou shi dao qiang. (Shi Nai'an The Outlaws of the Marsh Chapter 36)

Happy, with their mission, they gathered their weapons. (Translated by Shapiro)

Eg. 9 "Ya nei shi si xiang na shuang mu de, zhe cai ru he?" (Shi Nai'an The Outlaws of the Marsh Chapter 7)

"Lin Chong's wife. How is that for a guess?" (Translated by Shapiro)

Examples from 5 to 8 indicate that the Chinese idiom "huan tian xi di" has different translations in different contexts. The style is not what to be emphasized. If the meaning is expressed, translators can use various form to cope with the same structure. The reason for such a treatment is that the source text pays much attention to the meaning to be expressed rather than what style it takes. So, in order to achieve equivalence in function, varieties in style are possible only if the varieties can best suit the target language and also can achieve the same effect among the target readers. Example 9 demonstrates that the style of the original text has no identical structures in the target text. However, the 
source style has very deep and special meanings in the source text which can hardly be matched in the target text. "shuang mu" in Chinese makes another character "lin" which refers to "Lin Chong". English translation of such a structure is impossible to guarantee equivalence both in style and in meaning. In this case, a meaning oriented model is the most applicable selection, for one thing, it can to the largest extend match the meaning of the original text, and for another, it could be easily accepted by the target readers. Such selection actually is a passive one in that the translators have no other better choice to guarantee the closest equivalence between the target and source texts. To cast the original style basically is to protect the expression of the meaning.

\section{CONCLUSION}

The first conclusion to draw according to the above three models is that equivalence is the highest standard of translation. But however perfect the standard of equivalence is, it is only an ideal pursuit of the translators. Objectively, there are no absolute equivalent expressions even in the same language.

Another point to be noted is translation in nature concerns a process of selection. The original text contains many factors which would be typical in the source text, but unfortunately, such factors do not always have corresponding counterparts in the target text. Translators however, need to figure out which factor or factors should be and can be selected and then transferred to the target text. The selection should be considered reasonable if the selection can well be adaptable to the translation environment.

With the above two points, translators can well solve a big problem in translation - non-translatability. By nontranslatability, we mean the translators cannot find totally equal points in style, meaning and function. The translator's job, in this case is to figure out the closest match of the source text in the target text. Sometimes, to select means to cast for the sake of the highest equivalence. So we can conclude with safety that any text is translatable. The only difference between different translations lies in the degree of equivalence. The best translation is the best selection; the best translation is the one which is adaptable to the translation environment.

\section{REFERENCES}

[1] Halliday, M. A. K. (2000). An Introduction to Functional Grammar. Beijing: Foreign Language Teaching and Research Press.

[2] Hu Gengshen. (2009). Translation as Adaptation and Selection. Perspective: Studies in Translatology, 11 (4): 283-291

[3] Hu Gengshen. (2006). To Look at Translation Theory from the Translated Version. Foreign Language teaching (4) :50-54

[4] Hu Gengshen. (2006). Examples to Show the Principle and Methods of Translation. Foreign Languages and Their Teaching (3): 49-52.

[5] Jakobson. (1960). Linguistics and Poetics. Cambridge: MIT Press.

[6] Juliane House. (1977). A Model for Translation Quality Assessment: A Mode Revisited. Tübingen: Narr.

[7] Nida, E.A. (1964). Toward the Science of Translating. Leiden: Brill.

[8] Nida, E.A. (2001). Language and Culture: Contexts in Translation. Shanghai: Shanghai Foreign Language Education Press.

[9] Newmark, Peter. (1980). Approaches to Translation (Language Teaching Methodology Series). Phoenix ELT.

[10] Reiss, Kathariana. (1971). Possibilities and Limitations of Translation Criticism. Categories and Criteria for a Fair Evaluation of Translations. Manich: Hueber.

[11] Tytler, A.F. (1790). Essay on the Principles of Translation. Amsterdam: Benjamins.

[12] Wang Dongfeng. (2004).Deconstructing Loyalty-the End of the Myth of Translation. China Translation.

Guodong Zhao was born in FengZhen, Inner Mongolia in 1975. He got his Master's degree of Art at Foreign Languages College, Inner Mongolia University, Huhhot, Inner Mongolia, P. R. China, in 2008.

$\mathrm{He}$ is currently a lecturer in Foreign Languages Department of Inner Mongolia Finance and Economics College, Huhhot, Inner Mongolia, teaching Linguistics.

His major Publications include An Understanding of Pidgen from the Perspective of Chaos Theory at English Language Teaching, published by CCSE, Canada, June, 2010. His current research interest is Translation and linguistics.

Xiao Liu was Born in Bao Tou, Inner Mongolia in 1976, majoring in Linguistics. She got her Master's degree of Art at Foreign Languages College, Inner Mongolia University, Huhhot, Inner Mongolia, P. R. China, in 2008.

Now she is working as a lecturer in Foreign Languages Department of Inner Mongolia Finance and Economics College, Huhhot, Inner Mongolia, teaching English Literature. 